Original Article

\title{
Eliciting Requirements for a Diabetes Self-Management Application for Underserved Populations: A Multi-Stakeholder Analysis
}

Samuel Bonet-Olivencia ${ }^{1}$, Arjun H. Rao ${ }^{1}$, Alec Smith ${ }^{1}$, Farzan Sasangohar ${ }^{1,2^{*}}$

${ }^{1}$ Department of Industrial \& Systems Engineering, Texas A\&M University, College Station, TX

${ }^{2}$ Center for Outcomes Research, Houston Methodist, Houston, TX

*Correspondence: sasangohar@tamu.edu

\begin{abstract}
Medically underserved communities have limited access to effective disease management resources in the U.S. Mobile health applications (mHealth apps) offer patients a cost-effective way to monitor and self-manage their condition and to communicate with providers; however, current diabetes self-management apps have rarely included end-users from underserved communities in the design process. This research documents key stakeholder-driven design requirements for a diabetes selfmanagement app for medically underserved patients. Semi-structured survey-interviews were carried out with 97 patients with diabetes and 11 healthcare providers from medically underserved counties in South Texas to elicit perspectives and preferences regarding a diabetes self-management app, and their beliefs regarding such an app's usage and utility. Patients emphasized the need for accessible educational content and for quick access to guidance on regulating blood sugar, diet, and exercise and physical activity using multimedia rather than textual form. Healthcare providers indicated that glucose monitoring, educational content, and graphical visualization of diabetes data were among the top-rated app features. These findings suggest that specific design requirements for the underserved may improve the adoption, usability, and sustainability of such interventions. Designers should consider health literacy and numeracy, linguistic barriers, data visualization, data entry complexity, and information exchange capabilities.
\end{abstract}

Keywords: Diabetes mellitus; self-management; blood glucose self-monitoring; mobile applications; medically underserved area; health literacy; telemedicine; disease management 


\section{Introduction}

In 2020, over 30 million individuals in the United States suffered from diabetes, most (about 90\%) with type 2 diabetes [1]. Rural/medically underserved areas-defined as populations with low access to primary care providers, high infant mortality, high poverty, and/or high elderly population [2] - have shown relatively poor diabetes outcomes compared to the urban/well-served areas [3]. Additionally, Type 2 diabetes disproportionately affects people of certain racial and ethnic groups many of whom may live in areas identified as rural/medically underserved [4] such as Hispanics/Latinx Americans. Recent estimates from the Centers for Disease Control showed that individuals from Hispnic/Latinx American heritage were more likely (17\%) to suffer from diabetes than the non-Hispanic White population (8\%) [5]. Additionally, data from the U.S. Department of Health and Human Services' Office of Minority Health revealed that diabetes was among the leading causes for mortality among the non-White population [6].

Effective self-management of diabetes could have a significant impact on health outcomes. Studies have shown that patients who received training in self-management were successful in regulating their blood glucose levels, dietary habits, and glycemic control [7-11]. However, several barriers restrict the ability of underserved patients to execute self-management effectively [12]. These include limited access to timely healthcare services [13], limited financial resources [14], low literacy [15-17], and geographic barriers to seek care from providers outside their community [18]. Therefore, there is a need to investigate methods or interventions that enable self-management by identifying and addressing such barriers systematically.

Recent advances in mobile health (mHealth) technologies have shown promise in mitigating barriers related to accessibility. These technologies facilitate self-management of diabetes including discreet, costefficient, and non-invasive tools for monitoring health conditions, and a reliable platform for interactions between healthcare providers and patients $[19,20]$. While such characteristics make mHealth a promising method to address barriers to self-management in underserved populations, there is limited research documenting guidelines or $\mathrm{mHealth}$ design requirements for these populations $[21,22]$.

A recent review of 11 mHealth apps for diabetes [23] revealed common features such as setting reminders, tracking blood glucose and hemoglobin $\mathrm{A} 1 \mathrm{c}(\mathrm{HbA} 1 \mathrm{c})$, medication use, physical activity, and weight. However, usability tests on eight apps revealed that more than two-thirds (6/8) were scored by patients as "marginal" or "not acceptable" [23]. Another study [24] highlighted the lack of personalized feedback and usability issues including ease of data entry, and integration with patients and electronic health records suggesting a potential gap in user-centered design (UCD) approaches. UCD has shown promise in fostering user engagement and improving the perception of app effectiveness, with positive impact on sustained behavioral change $[25,26]$. To our knowledge, only a few attempts have been documented to utilize UCD to inform requirements for diabetes self-management apps (e.g., [27]) and no research has focused on needs and expectations of the underserved. To address this gap, in this paper, we document stakeholders' needs and expectations from a diabetes self-management app by eliciting feedback from diabetic patients and providers in several medically underserved areas in the United States.

\section{Methods}

Semi-structured interviews were conducted with a convenience sample of 97 patients and 11 healthcare providers from several medically underserved counties in South Texas. Interviews with patients were conducted by four nurse educators with graduate degrees in nursing or education during diabetes selfmanagement education sessions as part of the Healthy Texas initiative. Participants were informed about 
the study at the end of educational sessions and were selected if they met the participation criteria (aged $18+$ and had diabetes). The authors FS and AR who held doctoral degrees in Engineering and had extensive experience in qualitative research, provided these interviewers with training on conducting interviews. Providers were recruited and interviewed by the authors AR, SB, and FS during a diabetes education conference in South Texas. The research group used a booth at the conference exhibition room to recruit the participants. The study team did not establish a relationship with the participants prior to the study and no one other than the interviewers were present at the interview sessions. No potential participant refused or withdrew mid-study, and no repeated interviews were carried out.

Two interview protocols were developed for patients and providers, respectively, to reduce individual biases and assumptions and to standardize the interviews. Interviews with patients focused on understanding their expectations from a diabetes self-management app. Similarly, interviews with providers focused on their expectations from a self-management app for diabetes both from the patients' perspective and the type of information or interactions providers expected from such tool. The interviews took approximately 45 minutes for both patients and providers. Patients and providers received a \$25 or $\$ 50$ gift card, respectively, for participation. The Texas A\&M University Review Board reviewed and approved this study (IRB Protocol \#IRB2018-1503D) and all participants provided informed consent.

The interviews were audio recorded and no field notes were made by the interviewers during or after the interviews. A transcription service, Temi, was used to transcribe the audio recorded interviews preceding analysis [28]. Thematic analysis of the interviews was conducted by two coders (AS and AR) [29,30]. The two coders completed the following steps, separately and sequentially, and then met to discuss any discrepancies: code creation, initial coding, and focused coding. The thematic coding process entailed a deeper discussion of the themes and constructs that emerged from the analysis. After coming to a consensus, the themes were discussed with the other authors (SB and FS) and changes were made as necessary. MAXQDA 12 was used to complete the analysis [31]. AS and SB were doctoral students and had extensive experience in qualitative data analysis.

\section{Results}

\subsection{Demographics of Participants}

\subsubsection{Patient Demographics}

Table 1 presents key demographics of the patients. A total of 100 patients participated in the interviews. After cleaning the data, removing incomplete entries, a total of 97 interviews were analyzed. The average age of the participants was 56.07 (SD=13.10). A vast majority of participants were Hispanic or Latinx ( $90 \%$, 87/97). Most of the patients did not have a postsecondary degree, with $73.20 \%$ (71/97) of the respondents having either some college (no degree), a high school diploma or less. Approximately half the respondents $(50.51 \% ; 49 / 97)$ had a household income of less than $\$ 30,000$. About a fourth of participants reported not having medical insurance (24\%; $23 / 97)$.

Table 1: Demographic information of patients

\begin{tabular}{|l|l|l|l|}
\hline \multicolumn{2}{|l|}{ Characteristic } & $\begin{array}{l}\text { Number of } \\
\text { Respondents }\end{array}$ & Percentage \\
\hline Gender $(\mathbf{n}=\mathbf{9 7})$ & Female & 71 & \\
\hline & Male & 26 & 73.20 \\
\hline
\end{tabular}




\begin{tabular}{|l|l|l|l|}
\hline \multicolumn{2}{|l|}{ Characteristic } & $\begin{array}{l}\text { Number of } \\
\text { Respondents }\end{array}$ & Percentage \\
\hline Income (n=97) & Less than \$20,000 & 30 & \\
\hline & $\$ 20,000-\$ 30,000$ & 19 & 30.93 \\
\hline & $\$ 30,000-\$ 40,000$ & 11 & 19.59 \\
\hline & $\$ 40,000-\$ 50,000$ & 10 & 11.34 \\
\hline & $\$ 50,000-\$ 60,000$ & 4 & 10.31 \\
\hline & Above \$60,000 & 8 & 4.12 \\
\hline & Prefer not to answer & 15 & 8.25 \\
\hline & & & 15.46 \\
\hline Race (n=97) & White (non-Hispanic or Latinx) & 7 & \\
\hline & Hispanic or Latinx (White) & 61 & 7.22 \\
\hline & Hispanic or Latinx (non-White) & 26 & 62.89 \\
\hline & American Indian or Native & 2 & 26.80 \\
\hline & Two or more races & 1 & 2.06 \\
\hline & & & 1.03 \\
\hline Education (n=97) & Less than high school diploma & 16 & \\
\hline & High school diploma or GED & 29 & 16.50 \\
\hline & Some college, no degree & 26 & 29.90 \\
\hline & Associate Degree & 14 & 26.80 \\
\hline & Bachelor's Degree & 14.43 \\
\hline & Graduate or Professional Degree & 3 & 9.28 \\
\hline & & & 3.09 \\
\hline & &
\end{tabular}

\subsubsection{Healthcare Provider Demographics}

Eleven healthcare providers serving medically underserved communities in South Texas participated in the interviews. Table 2 presents key demographics about the healthcare providers interviewed. On average, the physicians sampled had nearly three decades (mean=28.86; SD=7.75; Range: 10 to 38) of experience in their current roles. Most participants (9/11) practiced family medicine, one practiced general medicine, and one was a pediatric nurse practitioner. Two participants held leadership roles (president/CEO) in their respective organizations.

Table 2: Demographic information of healthcare providers

\begin{tabular}{|c|c|c|c|}
\hline \multicolumn{2}{|c|}{ Characteristic } & $\begin{array}{l}\text { Number of } \\
\text { Respondents }\end{array}$ & Percentage \\
\hline \multicolumn{4}{|c|}{ Gender ( $n=11)$} \\
\hline & Female & 2 & 18.19 \\
\hline & Male & 9 & 81.81 \\
\hline \multicolumn{4}{|l|}{ Age $(n=11)$} \\
\hline & $45-54$ years & 1 & 9.09 \\
\hline & $55-64$ years & 5 & 45.45 \\
\hline & $65-74$ years & 5 & 45.45 \\
\hline \multicolumn{4}{|l|}{ Race $(n=11)$} \\
\hline & White (Non-Hispanic or Latinx) & 9 & 81.81 \\
\hline & Hispanic or Latinx (non-White) & 2 & 18.19 \\
\hline \multicolumn{4}{|c|}{ Nature of Experience $(\mathrm{n}=11)$} \\
\hline & Family Medicine/Practice & 9 & 81.81 \\
\hline & General Medicine & 1 & 9.09 \\
\hline
\end{tabular}




\begin{tabular}{|l|l|l|l|}
\hline \multicolumn{2}{|l|}{ Characteristic } & $\begin{array}{l}\text { Number of } \\
\text { Respondents }\end{array}$ & Percentage \\
\hline & Pediatric Nurse Practitioner & 1 & 9.09 \\
\hline
\end{tabular}

\subsection{Participant Interview Themes}

Patients were asked to specify features they desired in a diabetes self-management mobile app. A total of 97 participants responded to this question. Analysis of these responses resulted in five superordinate themes: (1) logging and tracking of blood sugar readings; (2) assistance with adopting a healthy lifestyle; (3) integration with healthcare system; (4) reminders and alerts; and (5) usability and non-invasiveness. Almost $20 \%$ of the respondents $(19.58 \% ; 19 / 97)$ indicated that they did not know what features they would expect in a diabetes self-management app.

Healthcare providers were asked a series of questions about features they believed would benefit their patients and would improve their practice. Analysis of the responses from 11 physicians resulted in five superordinate themes: (1) dietary logs; (2) patient diabetes education; (3) reminders and alerts; (4) information communication and presentation; and (5) patient-related challenges and barriers. These themes and associated subthemes are discussed below. The proportion of participants whose response is captured by a theme or subtheme is presented with percentage (\%) and counts ( $x x / X X)$. Some subtheme counts do not total $100 \%$ because some participants had responses in multiple subthemes.

\subsubsection{Functional Requirements Suggested by Patients}

\section{Logging and Tracking Blood Sugar Readings}

Almost a quarter $(24.74 \%$; $24 / 97)$ of the patients in our sample expressed the need to be able to track and log their blood sugar readings. Two prominent subthemes were identified from the interviews: (a) logging readings and (b) assistance and insights from the readings.

Logging Readings: This subtheme captures patients' desire for the app to help them log and recall their blood sugar readings. More than half of the patients (54.17\%; $13 / 24)$ who expected this feature also highlighted the need to trace back to previous readings to check their well-being.

"Just to be able to keep track of myself... or tracking my glucose... without having to write it down" - P12

Assistance \& Insights from the Readings: Some patients (16.67\%; 4/24) pointed out the need to understand what the entries mean. Specifically, they indicated the need for graphical interfaces to visualize trends of their readings. For example, "keeping track of history...so I can monitor for trends" (P19). Participants mentioned familiarity with similar visual trend such as activity and expected similar visualization for sugar levels. In addition, some of the patients mentioned that descriptive statistics about their parameters would be useful in managing their condition such as "the daily average, and the weekly average" (P09).

\section{Assistance with Adopting a Healthier Lifestyle}

About a third (34.02\%; 33/97) of the respondents indicated the need for assistance with managing their condition and adopting healthier choices and demonstrated a willingness to learn about tips and 
techniques to manage their diabetes. Specifically, their responses were categorized into three subthemes: (a) diet regulation; (b) health tips; and (c) fitness and physical activity.

Diet Regulation: This theme captures patients' desire to be provided with information on regulating their eating habits and food intake. Two thirds $(66.67 \% ; 22 / 33)$ of these participants wanted diet regulation features including access to a list of the types of foods they could consume to maintain glycemic control (e.g., foods they could consume to avoid hypoglycemia). Further, patients also wanted the app to help them construct and adhere to a diet plan. Finally, some patients (based on the diabetes education they had received) indicated that they could benefit from having a carbohydrate "tracker".

"Like maybe like a diet plan, things to do or not to do you know that can lower your sugars if they're high." - P21

"[...] and a list of dos' and do not food, you know, like a list, an actual list." - P07

"How many carbs, I can [eat], you know, in, um, like in the mornings [...or] at lunchtime I'll have a sandwich [...] I think that's one of the reasons my diabetes goes up. It scares me, you know, to eat a lot of carbs." - P16

Health Tips: Patients frequently (39.39\%; 13/33) mentioned the need to easily access health-related resources. Although there was interest in health resources in general, patients were particularly keen on accessing specific tips about nutrition. Participants also mentioned expecting prescriptive tips when presented with abnormal blood sugar values.

"There's a lot of things like for your heart and stuff [...] there's a lot of stuff out here that we eat and we're not supposed to because it's really damaging ourselves. So, you know some advice [...] give us something like that." - P11

"[...] to see, to measure if your sugar is high or low and to explain what things you can do to lower our sugar $[\ldots] "$ " $-P 08$

Fitness and Physical Activity: Several of these patients (15.15\%; 5/33) indicated that while there are several commercial apps for fitness and activity tracking, a fitness module integrated into the diabetes self-management app would be ideal, suggesting the perceived importance of the connection between physical activity and diabetes.

\section{Reminders and Alerts}

Some patients (11.34\%; 11/97) suggested timely alerts or reminders would help them adhere to their medication regimen. Two subthemes emerged from patient responses: (a) reminders \& scheduling, and (b) predictive capability.

Reminders \& Scheduling: Several of these patients (72.72\%; 8/11) highlighted their busy lifestyle as a reason for forgetting to monitor their blood sugar levels. In addition to being reminded to monitor their health, patients also suggested that a scheduling tool would help them keep track of their appointments. 
Predictive Capability: Some of these patients $(36.36 \% ; 4 / 11)$ responded that they would like predictive features such as early detection of warning signs and monitoring trends so they can mitigate any problems before they occur.

"[...] maybe signs to look for, like when you're going to have maybe an [hypoglycemia] episode, so like warning signs." - P39

\section{Integration with Healthcare System}

A few patients in our sample (3.09\%; 3/97) highlighted the need for their diabetes self-management program to be integrated into their overall care system. Patients desired easy communication of diabetic parameters and progress reports with their healthcare provider.

"[...] being able to send it to the doctor, or bring a recording of the reading. That way they could keep track of it." - P06

\section{Usability and Non-invasiveness}

Several patients $(13.4 \% ; 13 / 97)$ desired a system that would be easy to use and be non-invasive. This superordinate theme can be categorized into two subthemes: (a) usability; and (b) non-invasiveness.

Usability: Several patients raised concerns about their experiences with app usability $(46.15 \% ; 6 / 13)$ and expected an app that was reliable, accurate, and easy to use.

Non-invasiveness: This theme captures patients' desire for a method of reading sugar levels without having to prick themselves, as commonly required by most glucometers. Most of these patients (53.84\%; 7/13) were fatigued by the constant pricking for blood sugar measurement and desired an app that would display blood sugar readings (potentially from an implantable continuous glucose monitor).

"Like I said, a feature that would allow you to check your glucose level without [pricking], ..., I mean I don't know if they can make something like that without drawing your blood." $-P 14$

\subsubsection{Functional Requirements Suggested by Healthcare Providers}

\section{Dietary Logs}

Healthcare providers $(72.72 \% ; 8 / 11)$ highlighted the importance of a diet/food log to keep track of what patients are consuming and to have patients engage in their treatment. Healthcare providers also suggested that the app provide immediate feedback to the patients about the calorie density and quality of the food they are ingesting. However, providers cautioned against using food logs in clinical assessments as patients tended to be dishonest in their logs.

"So, I mean, if they want to write it down, that's fine [...] if you're assuming perfect compliance and honesty. But my experience is that most patients aren't completely honest with what they do. So, I guess in the ideal setting, a food log would be great. So, you can go, I see when you have that bowl of ice cream, you know, that wasn't Broccoli, you know, then food log could be really important. So, I guess we could change it." - SO1 


\begin{abstract}
"Food log with [...] input about calorie and everything else. So, it'd be two-way [...] Immediate feedback. Get pretty much immediate feedback. If they're going to go to the trouble of entering in all that food, they need to get, I don't want it just to be written down, you know, and just stored somewhere and they look it up. They're going to enter what they're going to eat in a food log. They need to get immediate feedback about the calories or, and this is on or off their diet or something like that." - SO8
\end{abstract}

One physician alluded to the use of image processing and machine learning techniques to analyze a photograph of a plate of food. The results of the analysis provide a breakdown of calorie content and nutritional value.

"I thought it would be fantastic if a person sets their meal, their plate down, they take a photo of it. And artificial intelligence calculates the, based on the size of the plate, I mean, [...], how much potatoes take up, how much the meat takes up. And it calculates [...]. We load the fat amount, the protein amount [but] I don't think they have that yet." - SO4

\title{
Patient Diabetes Education
}

Some providers $(27.27 \% ; 3 / 11)$ highlighted the low health literacy of patients they treated and encouraged the creation of an educational component in the diabetes self-management app for facilitating communication with educators.

"We have to give them the information... It's like a coach. This is the game plan... this is how you throw the ball and all that. [You have] repetition and they get better at it." - S08

\section{Reminders and Alerts}

Most providers $(72.72 \% ; 8 / 11)$ suggested that providing periodic reminders or alerts about multiple topics including ingesting medication, diet adherence, activity reminders, and appointments would benefit patients and help them in self-management.

"Self-management. So yeah, you get reminders. You got to do that for them. Probably about every two hours... you remind them about if your glucose is too high or too low... They could do a reading... to help them for self-management." - SO2

Providers also cautioned designers about the tendency for patients to develop alarm fatigue leading to ignored reminders, thus highlighting the need to remind or alert only when appropriate.

"[...] there are patients who may feel like this is getting a little [annoying], and you're going to have to see everybody [feels] a little intruded." - S08

"[...] when you start getting emails that are 12 different things on the same subject, you just start going through them and not reading them. And that's what we're seeing. They will gloss over them." - SO5

When discussing reminders, healthcare providers' responses could be categorized into two subthemes:

(a) medication intake recall; and (b) activity reminder 
Medication Intake Recall: Several providers (50\%; 4/8) indicated some of their patients had trouble recalling the nature and amount of medication taken in a specific period. Therefore, the healthcare providers believed that including a medication an easy to use and intuitive reminder feature in the app would remind patients about previously ingested or impending medication.

"Having that in the app, so they're documenting it [...] I think from a provider standpoint it would be great, but from the patient standpoint, we can't get them to write it down in a book. It would have to be very simple. Like they go in and click a button or two, you know, have their medications, already populated and they could just go in and go click, click, click." - SO7

Activity Reminder: A few of the healthcare providers (37.5\%; 3/8) suggested designing a feature that would help patients log their activities and remind them to exercise/stay active while giving the provider access to that information.

"[...] we need something to help them exercise on here and way of recording it. [Even though] those are already with Fitbit's and stuff, but that needs to be sent to the physician." - SO4

\section{Information Communication and Presentation}

Although healthcare providers encouraged open lines of communication with their patients, they highlighted key features relating to data communication and presentation such as synchronous and asynchronous communication and information presentation.

Synchronous Communication: Two providers $(18.2 \% ; 2 / 11)$ stressed the need for both providers and patients to be able to communicate and exchange information, even for a self-management tool.

"Is there one-way or two-way communication with this app? It could be two-way. It has to be two. If it's two-way, I'd feel comfortable. If it's only one-way, it's not worth it."

The majority of providers $(63.63 \%$; $7 / 11)$ believed that text messages could be a useful medium to communicate specific, personalized, and urgent messages or instructions to patients, while a few preferred a chat feature.

"[...] texts would be for urgent things like too high or too low [blood sugar]."

"[...] what I like and what I think a lot of the younger crowd would like, would be, that "chat." [...] You know, if you have questions, you're gonna chat"

Asynchronous Communication: Providers had mixed feelings about the use of voice notes as a means of communicating with patients. While some healthcare providers $(27.27 \% ; 3 / 11)$ believed that voice notes might be beneficial for patients who were visually challenged, the majority $(72.72 \% ; 6 / 11)$ were against the use of voice notes, citing issues with understanding patient accents and dialects.

"[...] for those with really poor eyesight, it's gonna have to be a voice [recording], in their language." - SO4

"But you know as well as I do, there's so many dialects [...] word accents. Sometimes you can't understand." - SO3 
Information Presentation: Most providers were highly supportive $(72.72 \% ; 8 / 11)$ of having graphs in the mobile app. However, they remarked that some patients in their communities had challenges in comprehending the information provided in graphs and would often require the healthcare providers to describe it to them. Providers emphasized the need for the graphs to be simple, easy to read, with clearly displayed limits, and intuitive ways to warn patients about abnormal sugar levels.

"[...] People respond visibly very easily using warning colors. Green, good, red, bad. The line where yours is. Pictures and graphs are great and probably better than texts." - S08

Some healthcare providers indicated that adding appropriate pictures might help patients understand, interpret, and potentially maintain glycemic control.

"[...] they see somebody happy; they know their blood sugars in a happy range. Uh, see some blood sugars, they, they maybe they can follow it on a chart day to day. Happy face here means they're in control. A sad face here means they're out of control." - SO4

"[...] every picture tells a story. I think they would like pictures. See where they were and where they're going." - SO9

Of the six healthcare providers who responded to this question, $50 \%(3 / 6)$ were cautiously optimistic about the use of tables and charts to communicate clinical data to patients, while the other half felt that the patients might be overwhelmed.

\section{Patient-related Challenges or Barriers}

When asked about the potential to implement a diabetes self-management app in a clinical setting, healthcare providers highlighted key barriers that could impede care. Their responses were classified into the following subthemes: (a) temporal and physical barriers; (b) privacy concerns; (c) lack of motivation; and (d) patient literacy levels.

Patient Literacy Levels: Several providers (45.45\%; 5/11) emphasized the diversity in education levels of the patients and questioned patients' ability to read and interpret graphs or other information on an application. Further, providers also raised concerns about patients' general readiness to use technology-based interventions, age-related usability barriers, and the language barriers.

"Well, like I said, the people I'm going to use it on are usually older people and those people didn't grow up with technology." - S10

"Most of my people speak Spanish or Spanglish." - SO2

Some providers $(36.36 \% ; 4 / 11)$ indicated that many of their patients may struggle with selfmanagement which may require them to visit the physician (in-person) to interpret their readings. This may in turn exacerbate issues related to access and geographical barriers. They suggested integrating the app with existing technologies such as telemonitoring or providing means of communicating relevant information to address this barrier.

"If they have to come to the office [...] to present the data, that's a barrier. If it can be like the telehealth telemonitoring it's transmitted and that's not a barrier for them." - SO4 


\section{"[...] Transportation is a big barrier to adopt something like this. [Because] they have to get to the office. They also have looking for rides and I'm in a neighborhood, lot of people walk to my place, well [those] people have to take a bus." - SO2}

Privacy Concerns: A few providers $(18.18 \% ; 2 / 11)$ cited privacy concerns, highlighting that patients might be unwilling to be monitored or reluctant to share data.

"I don't know if they'd be open to doing something like that. [...] most of them don't [...]. They don't want something intruding on their [...] autonomy I guess." - S10

Lack of Motivation: A few providers (18.18\%; 2/11) also indicated that some of the patients in underserved areas might lack motivation to adhere to their treatment. This concern in turn relates to sustained app use for effective adoption of a healthier lifestyle.

\section{Discussion}

Our qualitative investigation into the requirements for a diabetes self-management app provided rich data on the key features and functionalities for patient adoption and engagement. These data lend insights into the facilitators and barriers that may encourage or impede diabetic patients' sustained use of a self-management app. Although there has been research on the preferences of medically underserved patients [21,22], our study adds the multi-stakeholder perspective of providers in medically underserved communities.

Our findings are consistent with previous literature on essential features for a diabetes self-management app [32,33]. Evidence-based guidelines suggest that logging and tracking blood glucose levels are essential elements in diabetes management $[34,35]$. Complementing these guidelines, Chavez and colleagues stated that physical activity, nutrition, blood glucose testing, medication or insulin dosage, health feedback, and education were key diabetes management tasks [36]. Consistent with this literature, patients in our study highlighted the need for logging and monitoring blood glucose levels, tips about health lifestyle choices (e.g., exercise, nutrition), and reminders-all key basic features in a diabetes selfmanagement app [33]. In addition, patients also requested the creation of a schedule feature, which would likely help them track their medication intake.

Patients were particularly interested in accessing educational resources to help them better self-manage their condition. Although patients interviewed in the study were receiving diabetes education from members of Texas A\&M Healthy South Texas (TAMHST), they requested dietary tips and educational content, which would likely help them better manage their condition. This request for educational content was supported by the healthcare providers in our sample. This preference for educational material highlights a critical gap in existing apps, with education among the most underrepresented features $[22,32]$. Further, it is important to note that content should be provided at levels consistent with the educational background of the population (primarily high school level or less in our sample). It is imperative that the content provided is aligned to recommendations in the literature to overcome challenges and limitations related to patients' literacy levels. Design implications such as minimizing technical jargon [37-42], presenting simplified language into tangible units [37-40], explaining uncommon terms [37-40], aligning content to patients' cultural background [37-39], and implementing visual and audible features over the use of text [37-43], must be considered when creating the educational content to be integrated in the mobile app. Williams and Schroeder [22] go on to state that the use of video-based 
educational material could complement text-based content since Hispanics are among the major consumers of online multimedia content [44]. Additionally, the availability of educational content in multiple languages seems to be essential to overcome language barriers, especially in those underserved communities where patients with diabetes are predominantly non-English speakers.

Our findings showed that patients were partial to understanding and interpreting their diabetic parameters through graphs and visualization. Although healthcare providers were generally supportive of these media, they cautioned against complex displays. Healthcare providers cautioned about patients' literacy levels and tech literacy levels, questioning their ability to read graphs and information in the app. One of the healthcare providers suggested the use of colors and imagery to convey meaning and urgency, a view supported by the findings of Desai and colleagues, who suggested the use of a traffic light representation and facial emotions among possible visualizations of blood glucose forecasts [45]. Further, while some patients indicated that they would benefit from blood sugar forecasting capabilities in the app, research has shown that individuals with low numeracy might find it difficult to interpret the uncertainties associated with a forecast, consequently leading to disengagement [45]. Providing the user with step-by-step guidance (e.g., a welcome wizard) about the different screen or features in the app could help address this problem [46]. Additionally, research has shown that difficulties using a smartphone can also impact the use of mobile apps [47]. Therefore, emphasis should be given towards orienting users to the app when they seem to be newer adopters of smartphone technology.

Patients expressed interest in logging data and notes relating to their diabetes self-management. Healthcare providers also encouraged patients to log information, in the belief that it would keep patients engaged in their treatment. However, research has shown that a common reason for abandoning the use of apps is due the time required to enter data [48], which our providers also considered as an issue. Selfmanagement practices can be encouraged by reducing the burden of data entry, for instance through simplified interfaces with adjustable text icon sizes to cater to individuals with visual impairments [22]. Additionally, data entry tasks could be simplified by the integration of databases and auto-fill features which help to minimize the amount of information the patients must recall and enter. Also, both providers and patients highlighted the importance of synchronous and asynchronous communication capabilities; however, healthcare providers highlighted difficulties that could arise due to language barriers. Therefore, the availability of communication functions should integrate translators.

The synergistic interplay between remote patient monitoring (RPM) systems and smartphone apps can play a key role in assisted diabetes self-management. Patients and healthcare providers agreed that seamless integration of the app with their healthcare infrastructure may improve monitoring and managing diabetes. Studies have shown that RPM systems facilitate monitoring vital signs and allow for early detection of potentially hazardous health conditions, allowing time for provider intervention and preventing expensive hospital admissions [49]. For example, a clinician could track an insulin-dependent patient's blood sugar profile, identify hypoglycemic tendencies, and make the necessary changes to the patient's insulin dosages. In addition, a recent study demonstrated the potential of an RPM-facilitated diabetes management program, which incorporated evidence-based lifestyle interventions [50]. However, enthusiasm to adopt RPM in medically underserved communities should be tempered by patients' access to technology, user proficiency, and training requirements [51-53].

This work has certain limitations. First, our sample did not contain medically underserved patients beyond those residing in a single county in South Texas. Therefore, the results may not be generalizable to other medically underserved communities. A similar limitation applies to the clinicians who were interviewed 
in our study. To overcome this limitation, we recommend carrying out the same study in multiple medically underserved communities across Texas and the U.S. Next, our sample consisted of only those patients who were seeking diabetes education and care through TAMHST. It is conceivable that there are other diabetic patients in that region who may have additional design requirements or have faced additional barriers not represented here. Finally, a significant proportion of the patients indicated that they were not knowledgeable about smartphone or self-management apps, thereby failing to provide design features. Although this is an unfortunate scenario, it presents an opportunity to educate patients about the capabilities of mHealth in assisting with diabetes self-management. It is imperative that these patients be included in formative usability tests to gather their perspective on the app design.

\section{Conclusions}

This paper highlights key features and functional requirements for the design of a diabetes selfmanagement app tailored to the underserved community. Our interviews with patients and physicians in some representative underserved areas suggest that specific design requirements for the underserved may improve the adoption, usability, and sustainability of such interventions. Despite the prevalence of several self-management apps, the emergence of patient education as a desired feature suggests the need for designers to pay closer attention to patients' linguistic abilities and health literacy levels. Both patients and providers also strongly desired the use of appropriate visualizations of diabetes data. In this regard, we recommend further investigation into the types of visualizations that would facilitate easy interpretation of diabetes data. The use of simplified interfaces, adjustable icons, databases, and auto-fill features were identified to simplify information visualization, information recall and data entry tasks. The use of formative training and technology exposure were identified to address issues of low experience with technology and low knowledge about mHealth capabilities, which could affect adoption and sustained engagement. Results from our study also provided insights into perceived patient adoption barriers including health literacy levels, motivation, and privacy concerns. To mitigate these barriers, we recommend adopting a community-based participatory research approach to facilitate a grassroots-level education about the capabilities of the app being designed.

\section{Authors Contributions:}

Conceptualization, F.S.; methodology, F.S., A.R. and S.B.O.; data collection, F.S., A.R. and S.B.O; formal analysis, A.R. and A.S.; writing-original draft preparation, A.R and A.S.; writing-review and editing, F.S. and S.B.O. All authors have read and agreed to the published version of the manuscript.

\section{Funding:}

This research was funded partly by the Robert J. Kleberg and Helen C. Kleberg Foundation and the National Science Foundation under Grant No. 1648451.

\section{Institutional Review Board Statement:}

The study was conducted according to the guidelines of the Declaration of Helsinki, and approved by the Texas A\&M University Review Board (IRB Protocol \#IRB2018-1503D).

\section{Informed Consent Statement:}

Informed consent was obtained from all subjects involved in the study. 


\section{Data Availability Statement:}

The data that support the findings of this study are available from the corresponding author, FS, upon reasonable request.

\section{Acknowledgements:}

We thank Jacob M. Kolman, MA, research associate at Texas A\&M University and scientific writer at Houston Methodist, for critical review and editing.

\section{Conflict of Interest:}

None declared.

\section{References}

1. Centers for Disease Control and Prevention. National Diabetes Statistics Report. 2020. Available online: https://www.cdc.gov/diabetes/pdfs/data/statistics/national-diabetes-statisticsreport.pdf (accessed on 18 October, 2021).

2. Health Resources and Services Administration. U.S. Department of Health and Human Services. MUA Find. 2021. Available online: https://data.hrsa.gov/tools/shortage-area/mua-find (accessed on 21 October, 2021).

3. Nichols, G. A.; McBurnie, M.; Paul, L.; Potter, J. E.; McCann, S.; Mayer, K.; Melgar, G.; D’Amato, S.; DeVoe, J. E. Peer reviewed: the high prevalence of diabetes in a large cohort of patients drawn from safety net clinics. Prev. Chronic Dis 2016, 13. PMID: 27309415

4. AuYoung, M.; Moin, T.; Richardson, C. R.; Damschroder, L. J. The diabetes prevention program for underserved populations: a brief review of strategies in the real world. Diabetes Spectr 2019, 32, 312-317. PMID: 31798288

5. Centers for Disease Control and Prevention. Hispanic/Latino Americans and Type 2 Diabetes. 2019. https://www.cdc.gov/diabetes/library/features/hispanic-diabetes.html (accessed on 18 October, 2021).

6. Kochanek, K.D.; Murphy, S.L.; Xu, J.; Arias, E. Deaths: final data. Nat/ Vital Stat Rep 2019, 68, 177. PMID: 32501199

7. Lirussi, F. The global challenge of type 2 diabetes and the strategies for response in ethnic minority groups. Diabetes Metab Res Rev 2010, 26, 421-32. PMID: 20641140

8. Lorig, K.R.; Holman, H. Self-management education: history, definition, outcomes, and mechanisms. Ann Behav Med 2003, 26, 1-7. PMID: 12867348

9. Lorig, K.R.; Ritter, P.L.; González, V.M. Hispanic chronic disease self-management: a randomized community-based outcome trial. Nurs Res 2003, 52, 361-9. PMID: 14639082 
10. Norris, S.L.; Engelgau, M.M.; Narayan, K.M. Effectiveness of self-management training in type 2 diabetes: a systematic review of randomized controlled trials. Diabetes Care 2001, 24, 561-87. PMID: 11289485

11. Reyes, J.; Tripp-Reimer, T.; Parker. E.; Muller, B.; Laroche, H. Factors influencing diabetes selfmanagement among medically underserved patients with type II diabetes. Glob Qual Nurs Res 2017, 4, 1-13. PMID: 28660239

12. Heisler, M.; Faul, J.D.; Hayward, R.A.; Langa, K.M.; Blaum, C.; Weir, D. Mechanisms for racial and ethnic disparities in glycemic control in middle-aged and older Americans in the health and retirement study. Arch Intern Med 2007, 167, 1853-60. PMID: 17893306

13. Rural Health Information Hub. Chronic Disease in Rural America. 2019. https://www.ruralhealthinfo.org/topics/chronic-disease (accessed on 18 October, 2021).

14. Piette, J.D.; Wagner, T.H.; Potter, M.B.; Schillinger, D. Health insurance status, cost-related medication underuse, and outcomes among diabetes patients in three systems of care. Med Care 2004, 42, 102-9. PMID: 14734946

15. Rothman, R.; Malone, R.; Bryant, B.; Horlen, C.; DeWalt, D.; Pignone, M. The relationship between literacy and glycemic control in a diabetes disease-management program. Diabetes Educ 2004, 30, 263-73. PMID: 15095516

16. Schillinger, D.; Barton, L.R.; Karter, A.J.; Wang, F.; Adler, N. Does literacy mediate the relationship between education and health outcomes? A study of a low-income population with diabetes. Public Health Rep 2006, 121, 245-54. PMID: 16640146

17. Schillinger, D.; Grumbach, K.; Piette, J.; Wang, F.; Osmond, D.; Daher, C.; Palacios, J.; DiazSullivan, G.; Bindman, A.B. Association of health literacy with diabetes outcomes. J Am Med Assoc 2002, 288, 475-82. PMID: 12132978

18. Douthit, N.; Kiv, S.; Dwolatzky, T.; Biswas, S. Exposing some important barriers to health care access in the rural USA. Public Health 2015, 129, 611-20. PMID: 26025176

19. Klonoff, D.C. The current status of mHealth for diabetes: will it be the next big thing? J Diabetes Sci Technol 2013, 7, 749-58. PMID: 23759409

20. Kitsiou, S.; Paré, G.; Jaana, M.; Gerber, B. Effectiveness of mHealth interventions for patients with diabetes: An overview of systematic reviews. PloS One 2017, 12. PMID: 28249025

21. Burner, E.R.; Menchine, M.D.; Kubicek, K.; Robles, M.; Arora, S. Perceptions of successful cues to action and opportunities to augment behavioral triggers in diabetes self-management: qualitative analysis of a mobile intervention for low-income Latinos with diabetes. J Med Internet Res 2014, 16. PMID: 24476784

22. Williams, J.P.; Schroeder, D. Popular glucose tracking apps and use of mHealth by Latinos with diabetes: review. JMIR MHealth Uhealth 2015, 3. PMID: 26307533 
23. Veazie, S.; Winchell, K,; Gilbert, J.; Paynter, R.; Ivlev, I.; Eden, K.B.; Nussbaum, K.; Weiskopf, N.; Guise, J.M.;Helfand, M. Rapid evidence review of mobile applications for self-management of diabetes. J Gen Intern Med 2018, 33, 1167-76. PMID: 29740786

24. El-Gayar, O.; Timsina, P.; Nawar, N.; Eid, W. Mobile applications for diabetes self-management: status and potential. J Diabetes Sci Technol 2013, 7, 247-62. PMID: 23439183

25. Cafazzo, J.A.; Leonard, K.; Easty, A.C.; Rossos, P.G.; Chan, C.T. The user-centered approach in the development of a complex hospital-at-home intervention. Stud Health Technol Inform 2009, 143, 328-33. PMID: 19380956

26. McCurdie, T.; Taneva, S.; Casselman, M.; Yeung, M.; McDaniel, C.; Ho, W., Cafazzo, J. mHealth consumer apps: the case for user-centered design. Biomed Instrum Technol 2012, 46, 49-56. PMID: 23039777

27. Goyal, S.; Morita, P.; Lewis, G.F.; Yu, C.; Seto, E., Cafazzo J.A. The systematic design of a behavioural mobile health application for the self-management of type 2 diabetes. Can J Diabetes 2016, 40, 95-104. PMID: 26455762

28. Temi. Audio to Text Automatic Transcription Service \& App [Internet]. 2020. https://www.temi.com/ (accessed on 21 October, 2019).

29. Guest, G.; MacQueen, K.M.; Namey, E.E. Applied Thematic Analysis. Sage Publications: Thousand Oaks, California, 2012. ISBN:9781483384436

30. Braun, V.; Clarke, V. Using thematic analysis in psychology. Qual Res Psychol 2006, 3, 77-101. doi:10.1191/1478088706qp063oa

31. VERBI Software. MAXQDA [software]. VERBI Software GmbH, Berlin, Germany, 2020.

32. Chomutare, T.; Fernandez-Luque, L.; Arsand, E.; Hartvigsen, G. Features of mobile diabetes applications: review of the literature and analysis of current applications compared against evidence-based guidelines. J Med Internet Res 2011, 13. PMID: 21979293

33. Goyal, S.; Cafazzo, J.A. Mobile phone health apps for diabetes management: current evidence and future developments. QJM Int J Med 2013, 106, 1067-9. PMID: 24106313

34. American Diabetes Association. Introduction: standards of medical care in diabetes-2018. Diabetes Care 2018;41(Supplement 1):S1-2. PMID: 29222369

35. National Institute for Health and Care Excellence. Type 2 diabetes in adults: management. 2020. https://www.nice.org.uk/guidance/ng28 (accessed on 18 October, 2021).

36. Chavez, S.; Fedele, D.; Guo, Y.; Bernier, A.; Smith, M.; Warnick, J.; Modave, F. Mobile apps for the management of diabetes. Diabetes Care 2017, 40, 145-6. PMID: 28774944

37. Hill-Briggs, F.; Smith, A. S. Evaluation of diabetes and cardiovascular disease print patient education materials for use with low-health literate populations. Diabetes Care 2008, 31, 667671. 
38. Centers for Disease Control and Prevention. Scientific and Technical Information Simply Put. 2nd ed. Centers for Disease Control and Prevention: Atlanta, Georgia, 1999.

39. Doak, C. C.; Doak, L. G.; Root, J. H. (1996). Teaching patients with low literacy skills. Am J Nurs 1996, 96.

40. National Cancer Institute. Clear and Simple: Developing Effective Print Materials for Low-Literate Readers. U.S. Department of Health and Human Services: Bethesda, MD, 1994.

41. Huang, Y. M.; Shiyanbola, O. O.; Chan, H. Y. A path model linking health literacy, medication selfefficacy, medication adherence, and glycemic control. Patient Educ Couns 2018, 101, $1906-$ 1913.

42. Osborn, C. Y.; Cavanaugh, K.; Kripalani, S. Strategies to address low health literacy and numeracy in diabetes. Clin Diabetes 2010, 28, 171-175.

43. Carstens, A. Tailoring print materials to match literacy levels: A challenge for document designers and practitioners in adult literacy. Language Matters 2004, 35, 459-484.

44. Google/OTX. Four Truths about US Hispanic Consumers. 2010. http://www.gstatic.com/ads/research/en/2010 FourTruthsAboutUSHispanics.pdf (accessed 18 October 2021).

45. Desai, P.M.; Levine, M. E.; Albers, D. J.; Mamykina, L. Pictures worth a thousand words: reflections on visualizing personal blood glucose forecasts for individuals with type 2 diabetes. $\mathrm{CHI}$ '18: Proceedings of the $2018 \mathrm{CHI}$ Conference on Human Factors in Computing Systems; 2018; Paper No. 538, pp. 1-13.

46. Isaković, M.; Sedlar, U.; Volk, M.; Bešter, J. Usability pitfalls of diabetes mHealth apps for the elderly. J Diabetes Res 2016, 1-9. PMID: 27034957

47. Skrøvseth, S.O.; Årsand, E.; Godtliebsen, F.; Hartvigsen, G. Mobile phone-based pattern recognition and data analysis for patients with type 1 diabetes. Diabetes Technol Ther 2012, 14, 1098-104. PMID: 23035775

48. Brzan, P.P.; Rotman, E.; Pajnkihar, M.; Klanjsek, P. Mobile applications for control and self management of diabetes: a systematic review. J Med Syst 2016, 40, 210. PMID: 27520615

49. Simons, D.; Egami, T.; Perry, J. Remote patient monitoring solutions. In Advances in Health Care Technology Care Shaping the Future of Medical Care; Spekowius, G.; Wendler, T. Springer Netherlands: Dordrecht, 2006; pp. 505-16. ISBN: 978-1-4020-4384-0

50. Michaud, T.L.; Siahpush, M.; Estabrooks, P.; Schwab, R.J.; LeVan, T.D.; Grimm, B.; Ramos, A.K.; Johansson, P.; Scoggins, D.; Su, D. Association between weight loss and glycemic outcomes: a post hoc analysis of a remote patient monitoring program for diabetes management. Telemed $E$ Health 2019, 26, 621-628. PMID: 31411552 
51. Alvarado, M.M.; Kum, H.C.; Coronado, K.G.; Foster, M.J., Ortega, P.; Lawley, M.A. Barriers to remote health interventions for type 2 diabetes: a systematic review and proposed classification scheme. J Med Internet Res 2017, 19, e28. PMID: 28193598

52. Christodoulakis, C.; Asgarian, A.; Easterbrook, S. Barriers to adoption of information technology in healthcare. Proceedings of the 27th Annual International Conference on Computer Science and Software Engineering, Ontario, Canada, 6-8 Nov 2017. Available online:

http://www.cs.toronto.edu/ christina/documents/ACM CASCON2017.pdf (accessed 18 October 2021).

53. Moiduddin, A.; Moore, J. The underserved and health information technology - issues and opportunities. Office of the Assistant Secretary for Planning and Evaluation (ASPE), U.S. Department of Health and Human Services; 2008. Available online:

https://aspe.hhs.gov/system/files/pdf/134261/report.pdf (accessed 18 October 2021). 